\title{
Correction to: Interference-free photoelectrochemical immunoassays using carboxymethylated dextran-coated and gold-modified TiO2 nanotube arrays
}

\author{
Wanze Guo ${ }^{1} \cdot$ Jinping Wang ${ }^{1} \cdot$ Wenjuan Guo ${ }^{1} \cdot$ Qing Kang ${ }^{1} \cdot$ Feimeng Zhou ${ }^{1}$ \\ Received: 14 July 2021 / Accepted: 14 July 2021 / Published online: 29 July 2021 \\ (C) Springer-Verlag GmbH Germany, part of Springer Nature 2021
}

\section{Correction to: Analytical and Bioanalytical Chemistry https://doi.org/10.1007/s00216-021-03442-0}

The authors would like to call the reader's attention to the fact that, unfortunately, there was an error regarding the text and respective legend of Fig. 5B and 5C.

Please find the correct version below. These corrections have no effect on any other results published in the article, and do not affect the discussion or any of the published conclusions.

As mentioned in the Introduction, regenerable electrodes can enhance sample throughput and improve measurement reliability over electrodes that can only be used once or twice. We found that our electrode surface can be regenerated at least five times. As shown in Fig. 5A, the photocurrent changes of five consecutive assays of an $8.80-\mathrm{pM}$ cTnI solution are all less than $10 \%$ of the average. After the sixth regeneration, the photocurrent change exceeded $11.7 \%$. While the immunosensor of anti-cTnI/N-acetyl-L-cysteine-CdAgTe quantum
dots/AuNPs/glassy carbon can be regenerated only one time by soaking in $5 \mathrm{M}$ carbamide solution, which maintained $95 \%$ and $87 \%$ of its original photocurrent after two and three exposures to $4.40-\mathrm{pM}$ cTnI, respectively [9]. Moreover, as mentioned in the Introduction, CMdextran films are advantageous in that they can effectively prevent surface fouling or nonspecific adsorption of biomolecules. At the $\mathrm{Au} / \mathrm{TiO}_{2}$ NTA (i.e. without the CMdextran coating), the first incubation of the electrode in a serum sample resulted in a decrease of photocurrent from $9.49 \mu \mathrm{A}$ (black curve in Fig. 5B) to $8.07 \mu \mathrm{A}$ (red curve), a net change by $15 \%$. The second incubation further decreased the photocurrent to $7.94 \mu \mathrm{A}$ (blue curve; $~ 16.4 \%$ decrease), suggesting that nonspecific adsorption of species from the serum was serious and had passivated the entire sensor surface. By contrast, three consecutive detections of cTnI in a serum sample led to a cumulative decrease by only $1.9 \%$ at our CM-dextran $/ \mathrm{Au} / \mathrm{TiO}_{2} \mathrm{NTA}$ (Fig. 5C). As a hydrogel, dextran is much more effective than many surface modifiers in preventing fouling of

The online version of the original article can be found at https://doi.org/ $10.1007 / \mathrm{s} 00216-021-03442-0$

Jinping Wang

ila_wangjp@ujn.edu.cn

Qing Kang

ila_kangq@ujn.edu.cn

1 Institute of Surface Analysis and Chemical Biology, University of Jinan, Jinan 250022, Shandong, China 

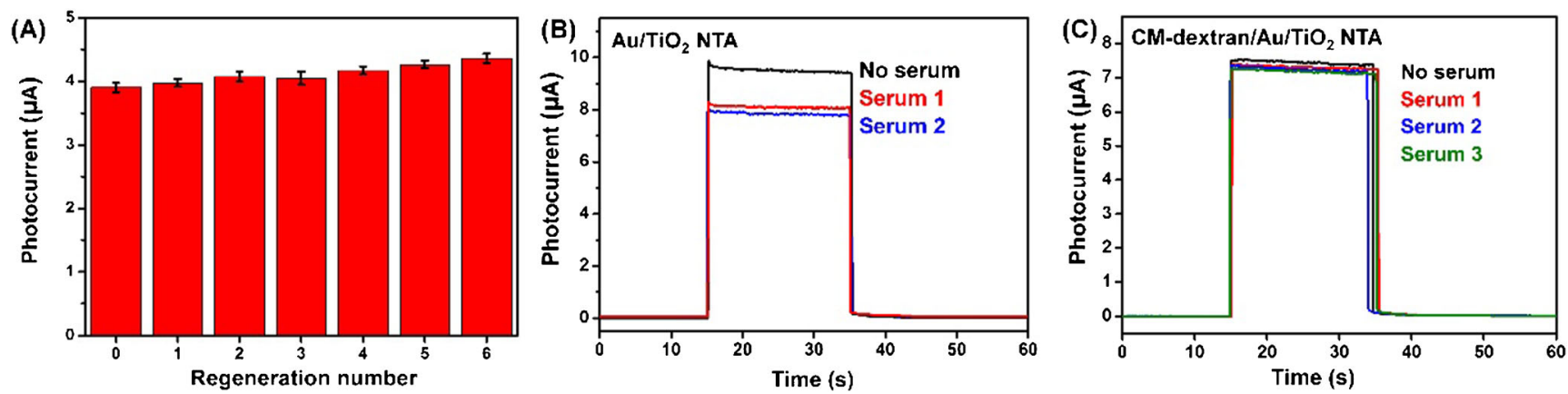

Fig. 5 (A) Photocurrent variations with assay numbers. (B) Chronoamperometric responses at an $\mathrm{Au} / \mathrm{TiO}_{2} \mathrm{NTA}_{\text {and }}(\mathbf{C})$ a $\mathrm{CM}-\mathrm{dextran} / \mathrm{Au} / \mathrm{TiO} \mathrm{O}_{2} \mathrm{NTA}$ before (black curves) and after exposures to and measurements of $\mathrm{cTnI}$ in three serum samples (red, blue, and green)

surface by interfering adsorbates. Pre-immobilization of CM-dextran onto the AuNPs-modified $\mathrm{TiO}_{2}$ NTA paved the way for our immunoassays of cTnI in serum samples described below.
Publisher's note Springer Nature remains neutral with regard to jurisdictional claims in published maps and institutional affiliations. 